\title{
AVALIAÇÃO DA QUALIDADE DE VIDA EM PORTADORES DE CÂNCER DE TIREÓIDE ATENDIDOS NA UNIDADE DE ALTA COMPLEXIDADE EM ONCOLOGIA (UNACON) E MULTICLIN DE FEIRA DE SANTANA-BA
}

\section{Jaíne Rocha e Silva ${ }^{1}$; Márcio Campos Oliveira² ${ }^{2}$ Marla Smille Pedrosa Cruz Ribeiro $^{3}$ e Tércio Guimarães Reis ${ }^{4}$}

1. Bolsista PIBIC/CNPq, Graduanda em Odontologia, Universidade Estadual de Feira de Santana, e-mail: jainerocha@outlook.com

2. Orientador, Departamento de Saúde, Universidade Estadual de Feira de Santana, e-mail: marciopatologiaoral@gmail.com

3. Participante da pesquisa, Doutoranda em Saúde Coletiva, Universidade Estadual de Feira de Santana, e-mail: m.smille@hotmail.com

4. Participante da pesquisa, Cirurgião de cabeça e pescoço, Unidade de de Feira de Santana, e-mail: tercioguimaraes@icloud.com

PALAVRAS-CHAVE: qualidade de vida; câncer de cabeça e pescoço; câncer de tireóide

\section{INTRODUÇÃO}

Segundo as estimativas da Organização Mundial da Saúde (OMS), a incidência de câncer na população mundial em 2030 será de 27 milhões de casos novos e o número de mortes por esta doença alcançará 17 milhões anualmente (INCA, 2011). O câncer de tireoide representa $5 \%$ de todos os casos de câncer diagnosticados no mundo (POLLOCK et al, 2006).

Essa doença é a neoplasia maligna mais comum do sistema endócrino, afetando mais frequentemente as mulheres do que os homens, sendo que a maioria dos casos ocorre entre pessoas de 25 a 65 anos de idade (ALMEIDA et al, 2013).

As consequências desse tipo de neoplasia podem afetar profundamente a Qualidade de Vida (QV) das pessoas acometidas, pois o tratamento é agressivo (mutilador), mesmo anos após o tratamento, interferindo nas atividades da vida diária, no que se refere à funcionalidade e à autoimagem (ONAKOYA et al, 2006).

Durante os últimos anos, a avaliação da qualidade de vida foi reconhecida como um importante medidor de sobrevida em medicina, sobretudo em oncologia. Nesse sentido, em 1993, o World Health Organization - Quality of Life Group (WHOQOL), definiu qualidade de vida como: percepção individual da posição do indivíduo na vida, no contexto de sua cultura e sistema de valores nos quais ele está inserido e em relação aos seus objetivos, expectativas, padrões e preocupações.

Diante de tal cenário, efetuar pesquisas sobre qualidade de vida em pacientes com câncer é fundamental para levantar os domínios afetados e planejar intervenções para reabilitar esses pacientes. O objetivo deste trabalho foi avaliar a qualidade de 
vida dos portadores de câncer de tireóide atendidos em unidades de referência em oncologia de Feira de Santana-Ba.

\section{MATERIAL E MÉTODOS OU METODOLOGIA}

O presente estudo foi realizado na Unidade de Alta Complexidade em Oncologia (UNACON) e Multiclin de Feira de Santana, BA. A população estudada foi constituída de pacientes portadores de câncer de tireoide em tratamento nas mais variadas modalidades, no período compreendido entre setembro de 2017 e julho de 2018. Foram incluídos na pesquisa todos os pacientes portadores de câncer de tireoide atendidos na UNACON e Multiclin de Feira de Santana - BA, que tiveram resultado histopalógico de câncer, idade igual ou superior a 18 anos e que aceitaram participar do estudo.

O procedimento de coleta de dados foi realizado apenas por uma pesquisadora previamente treinada e calibrada para tal procedimento e processamento dos dados obtidos. Os instrumentos de coleta dos dados consistiram de um questionário de Avaliação de Qualidade de Vida da Universidade de Washington (UW-QOL) em pacientes portadores de neoplasias malignas de cabeça e pescoço, devidamente validado para o idioma português. Neste questionário foram avaliadas as condições de saúde geral do paciente, atividade, recreação, dor, humor e ansiedade relativas à doença, e de um questionário sociodemográfico contendo dados relativos à idade, sexo, raça, renda e etc. Os dados foram avaliados descritivamente por meio da apresentação das frequências absolutas e percentuais relativas. O programa utilizado para a compilação dos dados foi o Statistical Package for Social Science - SSPS versão 10.0.

\section{RESULTADOS E/OU DISCUSSÃO}

Foram avaliados 19 pacientes com câncer de tireóide, sendo 17 mulheres e 2 homens correspondendo a $87,5 \%$ e $12,5 \%$ da amostra respectivamente. A faixa etária variou entre a terceira e a oitava décadas de vida, sendo 33 anos a menor idade e 87 anos a maior. Quanto à cor da pele 73,7\% dos pacientes se auto declararam pardos, a maioria se declarou solteiro. Quanto ao grau de instrução, apenas 5,3\% da amostra era analfabeta. Em relação a exposição solar 52,6\% se expunham frequentemente ao sol. Mais da metade da amostra não se encontra economicamente ativa e 31,6\% vivem com renda inferior a um salário mínimo. Quanto aos hábitos de vida, 73,7\% dos pacientes eram não fumantes e não etilistas. Dentre os doze domínios do questionário UW-QOL, as variáveis que apresentaram o menor escore foram: ansiedade seguida pelo humor. 
A qualidade de vida global dos pacientes foi analisada somando-se a pontuação de cada domínio do questionário UW-QOL (escore total), o que, portanto, pode variar de 0 a 1200, já que são 12 domínios, cada um podendo ser pontuado de 0 a 100. A média encontrada foi de 862,7 pontos. O paciente com a melhor qualidade de vida pontuou 1142 pontos, enquanto que o paciente com a pior qualidade de vida pontuou 725 pontos.

No presente estudo foi observada uma prevalência do câncer de tireóide nas mulheres o que está de acordo com o trabalho realizado por Sampaio (2004) onde foi encontrada uma prevalência seis vezes maior para o sexo feminino. Quanto à idade foi possível observar uma maior ocorrência na faixa etária entre 33 e 62 anos, indo ao encontro do estudo realizado por Nunes (2011) em que 50\% dos casos apresentaram idade entre 35 e 65 anos. Entretanto, em um estudo realizado pela Sociedade Brasileira de Endocrinologia e metabologia (2006) verificou uma faixa etária de 20 a 65 anos.

Em relação ao estado civil a maioria da amostra não se encontra em nenhum tipo de união estável, indo de encontro ao estudo de Nunes ( 2011), em que 65,6\% da amostra se apresentava em relação conjugal. No que concerne ao nível de instrução, apenas $36,8 \%$ dos pacientes haviam completado o ensino médio. Segundo a literatura a baixa escolaridade pode colaborar para demora no diagnóstico da doença e na dificuldade de compreensão e sucesso do tratamento. Outro fator limitante é a renda, neste estudo observou-se que $31,6 \%$ dos pacientes viviam com menos de um salário mínimo (MAYBURY et al, 2012).

Quanto aos hábitos de vida, notou-se que $73,7 \%$ dos entrevistados não fizeram uso de tabaco ou álcool, o que é um fator positivo, visto que, segundo o INCA (2010), o uso concomitante do fumo e do álcool aumenta em até 100 vezes o risco de desenvolvimento de câncer na região de cabeça e pescoço, principalmente nas regiões de boca, faringe e laringe.

$\mathrm{Na}$ avaliação da qualidade de vida dos pacientes, não houve decréscimo significativo nos fatores físicos, o que pode estar relacionado ao excelente prognóstico da doença e a taxa de sobrevida após um ano de 93\% (PACINE et al, 2012; KAPLAN et al, 2013), esses resultados corroboram com um estudo realizado por Furino et al (2017), onde não houve diferença da qualidade de vida geral dos pacientes com câncer de tireóide em relação à população em geral.

\section{CONSIDERAÇÕES FINAIS}


A qualidade de vida é um aspecto subjetivo que envolve a consideração de aspectos sociais, ambientais e psicológicos. Neste estudo, a qualidade de vida dos pacientes portadores de câncer de tireóide não se mostrou significativamente afetada, entretanto o domínio ansiedade se mostrou bastante afetado, o que sugere que esses pacientes devem ter um acompanhamento psicológico a longo prazo e que mais estudos nessa área precisam ser realizados, a fim de proporcionar uma assistência multidisciplinar a essas pessoas.

\section{REFERÊNCIAS}

AlMEIDA, A.F; AlVES, R.C; FELIX, J.D; CASTRO, D.S, et al.Qualidade de Vida das Pessoas Acometidas por Câncer no Trato Aerodigestivo Superior em um Hospital Universitário. Revista Brasileira de Cancerologia 2013; 59(2): 229-237

SAMPAIO, N. Dados epidemiológicos de pacientes submetidos à Iodoterapia. 2004. Disponível em sbbmn.org.br/eventos/ccbmn/ resumos/aprovados.pdf acessado em 29 de julho de 2018

SBEM (2006). Sociedade Brasileira de Endocrinologia e Metabologia. Proteja-se do Câncer de Tireóide. Disponível em http://www.endocrino.org.br/. Acessado em 29 de julho de 2018.

FURINO, F.O; FURINO, V.O; MELO, D.G; AVO, R.L.S; MAION, V.H; GERMANO, C.M.R; Cogitare Enferm. (22)3: e52038, 2017.

INSTITUTO NACIONAL DE CANCER José Alencar Gomes da Silva. Estimativa 2012: incidência de câncer no Brasil [Internet]. Rio de Janeiro; 2011 [acesso 2017 mar 07]. Disponível em: http://www1.inca.gov.br/ estimativa/2012/tabela estados.asp?UF=BR

INCA (2010) Instituto Nacional de Câncer. (20 de dezembro de 2010). Página visitada em 29 de julho de 2018.

KAPLAN, S.P; CAO, H; WEISS, R.E; DEGROOT, L.J; SIMON, C.A; EMBIA, O.M; ANGELOS, P; KAPLAN, E.L; SCHECHTER, R.B. A study of recurrence and death from papillary thyroid cancer with 27 years of median follow-up. Surgery2013; 154(6) :1436-1446.

MAYBURY, C; HOROWITZ, A.M; GOODMAN, H.S. Outcomes of oral cancer early detection and prevention statewide model in Maryland. J Public Health Dent. 2012; 72 Suppl 1:S34-8. doi: 10.1111/j.1752- 7325.2012.00320.x.

PACINI, F; CASTAGNA, M.G; BRILLI, L; PENTHEROUDAKIS, G. Guidelines Working Group. Thyroid cancer: ESMO Clinical Practice Guidelines for diagnosis, treatmentand follow-up. Ann Oncol 2012; Supl. 7:vii110- 119 